\title{
Die Lokalkasus im Komi aus der Perspektive der kognitiven Grammatik
}

Николай Кузнецов: Пространственная семантика местных падежей коми языка (когнитивныцй анализ) [Räumliche Semantik der Lokalkasus im Komi (kognitive Analyse)]. Dissertationes philologiae uralicae Universitatis tartuensis 12. Tartu 2012. $245 \mathrm{~S}$.

Nikolai Kuznecov analysiert in seiner Dissertation die Lokalkasus des Komi im Referenzrahmen der kognitiven Grammatik. Die Arbeit lässt die gründliche Vertrautheit des Verfassers mit der kognitiven Linguistik und seine guten muttersprachlichen Kenntnisse deutlich erkennen. Das Lokalkasussy- stem des Komi ist reichhaltig: es umfasst 12-13 Kasusformen, die in innere Lokalkasus (Inessiv - $b l t$, Elativ - bıcb und Illativ -ö), äußere Lokalkasus (Approximativ -лань, Egressiv -сянь und Terminativ -ӧд3), Prolativ (Prolativ I oder Prosekutiv -öd und Prolativ II oder Transitiv - $m i)$ sowie in die approximativen Lokalkasus (Approximativ-Inessiv -ланьын, Approximativ-Elativ -ланьысьь, Approximativ II -ланьӧ, Approximativ-Egressiv -ланьсянь, Approximativ-Prolativ -ланьӧд, -ланьті und Approximativ-Terminativ -ланьӧдз) gegliedert werden. (S. 88-89). Die letztgenannten, die approximativen Kasus, wurden erst in der großen 
komisprachigen Grammatik des Komi aus dem Jahr 2000 erstmals als Kasus klassifiziert. Sie werden äußerst selten verwendet und sind insofern marginal. (S. 165).

In seinem Überblick über die frühen Grammatiken des Komi schließt sich Kuznecov der heftigen Kritik an, die später an der ersten gedruckten Grammatik des Komi geübt wurde. Diese 1813 erschienene Grammatik von A. Fljorov überträgt u. a. das Kasussystem des Lateinischen unmittelbar auf das Komi (S. 34). Eine stärker kontextgebundene Perspektive wäre hier angebracht. Die frühen Grammatiken vieler Sprachen wurden nach dem Modell des Lateinischen verfasst und hatten offenkundig den Zweck, Menschen, die das Lateinische beherrschten, als Lehrmittel $\mathrm{zu}$ dienen. Das Lateinische stellte eine Art allgemeine Grammatiktheorie dar.

Kuznecov skizziert die grundlegende Gliederung der Lokalkasus sorgfältig. Die äußeren Lokalkasus bilden eigentlich keine Reihe und keine Entsprechung zu den inneren Lokalkasus, wie z. B. in den ostseefinnischen Sprachen die $l$-Kasus zu den $s$-Kasus. Die alten $l$-Kasus der permischen Sprachen haben keine spatiale Bedeutung mehr, sondern an ihrer Stelle werden in erster Linie Postpositionen verwendet. Interessanterweise hat sich aus den postpositionalen Ausdrücken in den Dialekten des Komipermjakischen eine neue Reihe von äußeren Lokalkasus mit $l$ - gebildet, da die Postpositionen lautlich abgeschliffen wurden und als Suffixe an die Wörter traten (S. 89-92). Obwohl die eigentliche Perspektive der vorliegenden Arbeit weder historisch noch vergleichend ist, bietet sie für weiterführende Untersuchungen viele Aspekte, die die synchronische und die diachronische Forschung verbinden. Auch bei der Erforschung des Komi ist die geringe Zahl der Korpusuntersuchungen offenkundig, worauf Kuznecov mehrfach hinweist. In dieser kognitiven Basisanalyse beschränkt Kuznecov sich auf ungefähre Berechnungen und auf seine Intuition z. B. hinsichtlich der Gebräuchlichkeit der verschiedenen Kasus in einer bestimmten Bedeutung. Eine tiefergehende Analyse würde zweifellos von statistischen Methoden profitieren.

Die Einführung in die kognitive Sprachforschung ist gründlich. Stellenweise werden recht triviale und in der Sprachwissenschaft (wie auch in anderen Bereichen) weithin verwendete Theorien mit Beispielen illustriert. An anderen Stellen werden auf höchst abstrakter Ebene angesiedelte Erklärungen eines Spezialbereichs der kognitiven Linguistik in keiner Weise veranschau- 
licht (z. B. S. 80 und 81). Zudem hätte die theoretische Grundlage sich stärker auf diejenigen Instrumente konzentrieren sollen, die bei der Behandlung der Lokalkasus des Komi in den späteren Kapiteln verwendet werden.

Der Text auf den Seiten 125-126, der die Lokalisation Domus behandelt, deckt sich weitgehend mit den Seiten 101-102. Solche Überlappungen hätten gestrichen werden sollen, ebenso die Wiederholung der Beispiele. Auch die Nummerierung der Beispiele wirkt befremdlich. Es wäre leserfreundlicher gewesen, sie für die Dissertation zu systematisieren. Man gewinnt den Eindruck, dass die ursprüngliche Nummerierung eines größeren Korpus unverändert beibehalten wurde.

Die Analyse der Kasus ist ein wenig uneinheitlich: Einige werden erkennbar gründlicher behandelt als andere. Kuznecov hatte zuvor in wissenschaftlichen Zeitschriften drei Beiträge über den Approximativ publiziert. Die Analyse dieses Kasus zählt denn auch zu den besten Teilen der Dissertation. Das dazu präsentierte Netzwerkmodell ist unverkennbar das Ergebnis langer Erwägung (S. 150). Ein wesentlicher Bestandteil der Bedeutung des Approximativs ist die Bewegung auf etwas zu, das jedoch nicht erreicht wird. Dennoch wird mit dem Approximativ manchmal auch das
Erreichen des Ziels ausgedrückt. Ein derartiger Widerspruch wäre aus der Sicht der traditionellen Grammatik schwer beschreibbar, doch die Methoden der kognitiven Grammatik erleichtern diese Aufgabe. Im Komi kann man den Ausdruck „auf das Zuhause zu“ oder „auf die Stadt zu“ verwenden, auch wenn aus dem Kontext hervorgeht, dass man tatsächlich nach Hause oder in die Stadt gelangt. Kuznecov liefert hierzu die vortreffliche Beobachtung, dass es sich in diesen Fällen um ein bewohntes Ziel handelt, das relative, unbestimmte Grenzen hat. (S. 147-148). Diese Unbestimmtheit ermöglicht die Deutung, dass das Ziel erreicht wird.

Auch die Darstellung des Prolativs ist gelungen. Der Prolativ wird in den permischen Sprachen häufig verwendet, und so ist auch seine zahlreiche periphere Verwendung erwartungsgemäß. Im Komi wird er nicht nur zum Ausdruck der Strecke und des Durchschreitens benutzt, sondern u.a. auch für den Schauplatz eines Ereignisses und für die Verbindungsstelle. (S. 136-138). Im Komi wird der Prolativ oft in Verbindung mit Bewegungsverben verwendet, um den Ort des Geschehens zu bezeichnen, worauf mit dem Terminus dynamische Lokalisation verwiesen wird (S. 140). Im Komi sagt man also 
Die Lokalkasus im Komi aus der Perspektive der kognitiven Grammatik

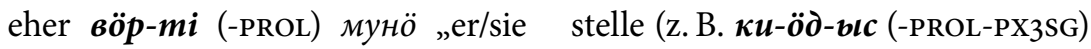
geht durch den Wald“ als „er/sie бocbmic ,er/sie nahm (ihn/sie) bei geht im Wald“, auch wenn sich das der Hand') von der Bedeutung Gehen ausschließlich im Wald ab- ,Durchgang ausgehend ausgesprospielt (S. 136). Gemeinsamkeiten der chen leicht zu erklären. (S. 138).

Grundbedeutungen des Prolativs Kuznecovs Dissertation ist ein sind die Fortbewegung und der Ort. willkommener und gelungener Es wäre eine interessante Frage für Neubeginn. Die kognitive Linguieine weiterführende Untersuchung, stik ist bei der Erforschung der östwelcher Bedeutungsunterschied lichen finnisch-ugrischen Sprachen im Komi entstünde, wenn anstelle bisher nur selten angewandt wordes Prolativs in solchen Fällen der den. Künftig kommt sie hoffentlich Inessiv verwendet würde. Auf der häufiger zur Anwendung.

Basis der kognitiven Linguistik ist die Bedeutung der Verbindungs- 